\title{
EXPLICIT BOUNDS FOR APPROXIMATION RATES OF BOUNDARY CROSSING PROBABILITIES FOR THE WIENER PROCESS
}

\author{
K. BOROVKOV, ${ }^{*}$ University of Melbourne \\ A. NOVIKOV,** University of Technology, Sydney
}

\begin{abstract}
We give explicit upper bounds for convergence rates when approximating both one- and two-sided general curvilinear boundary crossing probabilities for the Wiener process by similar probabilities for close boundaries of simpler form, for which computation of the boundary crossing probabilities is feasible. In particular, we partially generalize and improve results obtained by Pötzelberger and Wang in the case when the approximating boundaries are piecewise linear. Applications to barrier option pricing are also discussed.

Keywords: Wiener process; boundary crossing probability; barrier option

2000 Mathematics Subject Classification: Primary 60J65
\end{abstract}

Secondary 65C50; 91B70; 60G40

\section{Introduction and main results}

Computing the probability $P\left(g_{-}, g_{+}\right)$for the standard Wiener process $\left\{W_{t}\right\}_{t \geq 0}$ to stay within a corridor between two given boundaries $g_{-}(t)<g_{+}(t)$ during a specified time interval $[0, T]$ is crucial in many important applications, including sequential statistical analysis and the pricing of financial barrier options. In fact, based on the Donsker-Prokhorov invariance principle, such a probability is often used as an approximation to a similar boundary crossing probability for a random walk (or even a more general process). Computing the probability $P\left(g_{-}, g_{+}\right)$in nontrivial cases is, however, a rather tedious task by itself that, in its turn, also requires the use of some approximation methods.

One of the standard approachs to this problem is to approximate the given (general curvilinear) boundaries $g_{ \pm}$with some other boundaries $f_{ \pm}$, of a form enabling one to compute the probability $P\left(f_{-}, f_{+}\right)$relatively easily. A popular choice for $f_{ \pm}$is piecewise-linear boundaries, for which a combination of the total probability formula, the Markov property, and explicit formulae for linear boundary crossing probabilities for the Brownian bridge process immediately gives the desired probability $P\left(f_{-}, f_{+}\right)$as a finite-dimensional Gaussian integral of a product-form integrand [12], [14], [20]; also see our Remark 6, below. To justify the use of $P\left(f_{-}, f_{+}\right)$instead of $P\left(g_{-}, g_{+}\right)$, one must, of course, give an upper bound for the difference between the two values.

As a recent advance in this direction, we mention here a paper by Pötzelberger and Wang [14] (see also further references to be found in that paper). Those authors, under the assumptions that the boundaries $g_{ \pm}$are twice continuously differentiable, with $g_{ \pm}^{\prime \prime}(0) \neq 0$ and $g_{ \pm}^{\prime \prime}(t)=0$ at at most finitely many points $t \in(0, T]$, proposed a special rule for choosing a sequence of

\footnotetext{
* Postal address: Department of Mathematics and Statistics, University of Melbourne, Parkville 3010, Australia. Email address: kostya@ms.unimelb.edu.au

** Postal address: Department of Mathematical Sciences, University of Technology, Sydney, PO Box 123, Broadway, NSW 2007, Australia.
} 
'optimal partitions' $t_{0}^{(n)}=0<t_{1}^{(n)}<\cdots<t_{n}^{(n)}=T$ of $[0, T]$ (depending on the boundaries, in general) with the following property: if $g_{ \pm}^{(n)}$ are piecewise-linear boundaries with nodes at $\left(t_{i}^{(n)}, g_{ \pm}\left(t_{i}^{(n)}\right)\right), i=0,1, \ldots, n$, then for

$$
\Delta_{n}:=\left|P\left(g_{-}, g_{+}\right)-P\left(g_{-}^{(n)}, g_{+}^{(n)}\right)\right|,
$$

one has the asymptotic bound

$$
\limsup _{n \rightarrow \infty} n^{2} \Delta_{n} \leq A,
$$

where the constant $A$ depends on both the shape of the boundaries $g_{ \pm}$and the rule used to form the partitions $\left\{t_{i}^{(n)}\right\}_{0 \leq i \leq n}$ (through a pair of integrals that can actually be computed - at least numerically). Unfortunately, relation (1) does not contain much information about the approximation error size for finite values of $n$, although it is bounding that error for relatively small $n$ s (such that the computation of $P\left(g_{-}^{(n)}, g_{+}^{(n)}\right)$ is still feasible) that seems to be the main task here.

One could also observe that the above restrictions on the boundaries (in particular, on $g_{ \pm}^{\prime \prime}$ being nonzero) appear to be irrelevant (and are just due to the method employed in [14]). In this paper, we show that this is indeed the case, and that there is, in fact, an exact (rather than asymptotic, as in (1)) upper bound for $\Delta_{n}$ of the form $C n^{-2}$, which

(a) holds under more general assumptions on the boundaries $g_{ \pm}$; and

(b) holds for arbitrary partitions of $[0, T]$ of rank $O\left(n^{-1}\right)$.

(More generally, the upper bound has the form $C \delta^{2}$ for a partition of rank $\delta$ - see Corollary 1 , below).

This finding is based on a simple, general result that admits a short, self-contained proof, and of which a precise formulation is as follows. Let $g_{ \pm}(t)$ be two functions on $[0, T]$, such that $g_{-}(0)<0<g_{+}(0)$. Denote by

$$
P\left(g_{-}, g_{+}\right):=\mathrm{P}\left(g_{-}(t)<W_{t}<g_{+}(t), t \in[0, T]\right)
$$

the probability that the trajectory of the standard Wiener process $\left\{W_{t}\right\}_{t \geq 0}$ will stay between the boundaries $g_{ \pm}$during the whole time interval $[0, T]$. If $g_{-}(t) \geq g_{+}(t)$ at some $t \in[0, T]$, we simply get $P\left(g_{-}, g_{+}\right)=0$. In the case of a one-sided (upper) boundary, we will use the notation $P\left(-\infty, g_{+}\right)$.

By $\operatorname{Lip}_{K}$ we will denote the class of Lipschitz functions on $[0, T]$ with the constant $K \in$ $(0, \infty)$. That is, $g \in \operatorname{Lip}_{K}$ if and only if

$$
|g(t+h)-g(t)| \leq K h, \quad 0 \leq t<t+h \leq T .
$$

Also, by $\|\cdot\|$ we denote the uniform norm of a (bounded) function on $[0, T]$, i.e. $\|g\|=$ $\sup _{0 \leq t \leq T}|g(t)|$.

Theorem 1. Let $g_{ \pm} \in \operatorname{Lip}_{K}$ and consider functions $f_{ \pm}$on $[0, T]$ such that $\left\|g_{ \pm}-f_{ \pm}\right\| \leq \varepsilon$ for some $\varepsilon>0$. Then

$$
\left|P\left(-\infty, g_{+}\right)-P\left(-\infty, f_{+}\right)\right| \leq\left(2.5 K+2 T^{-1 / 2}\right) \varepsilon
$$

and

$$
\left|P\left(g_{-}, g_{+}\right)-P\left(f_{-}, f_{+}\right)\right| \leq\left(5 K+4 T^{-1 / 2}\right) \varepsilon .
$$


The same bounds also hold for the differences

$$
\left|P\left(-\infty, g_{+} ; B\right)-P\left(-\infty, f_{+} ; B\right)\right| \text { and }\left|P\left(g_{-}, g_{+} ; B\right)-P\left(f_{-}, f_{+} ; B\right)\right|,
$$

where, for a Borel set $B$,

$$
P\left(g_{-}, g_{+} ; B\right):=\mathrm{P}\left(g_{-}(t)<W_{t}<g_{+}(t), t \in[0, T] ; W_{T} \in B\right) .
$$

Remark 1. This result (or a weaker form thereof) might actually be already known. It was observed in Borovkov [4] that, with a right-hand side of the form $C\left(K+T^{-1 / 2}\right) \varepsilon$, where $C$ is some absolute constant, the above inequalities can be derived from Equation (2.22) of Nagaev [10], [11], estimates in Sahanenko [17] and the Donsker-Prokhorov invariance principle.

Remark 2. It is clear from the proof of Theorem 1 that a somewhat more precise bound than (3) holds in the two-sided boundary case: assuming that $g_{ \pm} \in \operatorname{Lip}_{K_{ \pm}}$, one can replace $5 K$ on the right-hand side of that bound with $2.5\left(K_{-}+K_{+}\right)$. Observe also that, under additional assumptions about the monotonicity of the boundaries $g_{ \pm}$, the values for the constants in bounds (2) and (3) can be made somewhat smaller (see Lemma 1, below).

Remark 3. Note that bounds (2) and (3) are rather sharp. Indeed, the coefficient of $\varepsilon$ on the right-hand side of (2) cannot be less than $2 K+(2 / \pi T)^{1 / 2}$. This can easily be seen from the explicit formula for $P\left(-\infty, g_{+}\right)$in the case of the straight line boundary $g_{+}(t)=\varepsilon+K t$ (cf. (14), below, and our observation immediately after that formula), when $f_{+}(t)=K t$, so that $P\left(-\infty, f_{+}\right)=0$.

Next we will formulate our improvement of (1), which is a simple consequence of Theorem 1 based on the fact that, for smooth enough functions, the rate of approximation by piecewiselinear functions will be a quadratic function of the partition rank. More precisely, the following result holds.

Corollary 1. Let $g_{ \pm}$be continuously differentiable on $[0, T]$, let $K=\max \left\{\left\|g_{-}^{\prime}\right\|,\left\|g_{+}^{\prime}\right\|\right\}$, and let $g_{ \pm}^{\prime}$ be absolutely continuous, satisfying $\left|g_{ \pm}^{\prime \prime}\right| \leq \gamma<\infty$ almost everywhere. If $0=t_{0}<$ $t_{1}<\cdots<t_{n}=T$ is a partition of $[0, T]$ of rank $\delta=\max _{0<i \leq n}\left|t_{i}-t_{i-1}\right|$, and $f_{ \pm}$are piecewise linear with nodes at the points $\left(t_{i}, g_{ \pm}\left(t_{i}\right)\right)$, then

$$
\left|P\left(-\infty, g_{+}\right)-P\left(-\infty, f_{+}\right)\right| \leq\left(0.313 K+0.25 T^{-1 / 2}\right) \gamma \delta^{2}
$$

and

$$
\left|P\left(g_{-}, g_{+}\right)-P\left(f_{-}, f_{+}\right)\right| \leq\left(0.625 K+0.5 T^{-1 / 2}\right) \gamma \delta^{2} .
$$

In particular, if the partition is uniform, i.e. $t_{i}=i T / n, 0 \leq i \leq n$, and if $g_{ \pm}^{(n)}$ denote the respective piecewise-linear approximations to $g_{ \pm}$, then $\delta=T / n$ and, hence, instead of the asymptotic bound (1), we obtain the following inequality:

$$
\Delta_{n} \leq C n^{-2}, \quad C=\left(0.625 K+0.5 T^{-1 / 2}\right) \gamma T^{2} .
$$

The same bounds will hold for the differences (4).

Remark 4. From the proof of the corollary, it is obvious that its assumptions can be somewhat relaxed: we need only assume that the boundaries $g_{ \pm}$are piecewise continuously differentiable (with the derivatives satisfying the stated conditions). The inequalities (5) and (6) remain valid as long as all those points at which either or both of $g_{ \pm}$is differentiable belong to the partition $\left\{t_{i}\right\}$. 
Remark 5. If the assumption that $g_{+}, f_{+} \in \operatorname{Lip}_{K}$ fails, but the functions are absolutely continuous with square-integrable derivatives and $g_{+}(0)-f_{+}(0)=g_{+}(T)-f_{+}(T)$, then the following bound holds:

$$
\left|P\left(-\infty, g_{+} ; B\right)-P\left(-\infty, f_{+} ; B\right)\right| \leq \mathrm{P}\left(W_{T} \in B\right)\left[\frac{1}{2 \pi} \int_{0}^{T}\left(g_{+}^{\prime}(s)-f_{+}^{\prime}(s)\right)^{2} \mathrm{~d} s\right]^{1 / 2} .
$$

The proof of this result, and similar bounds for the two-sided boundary crossing probabilities in the case $B=(x, \infty)$, were given in [12].

Remark 6. It appears that Wang and Pötzelberger [20] were the first to combine the total probability formula, the Markov property of the Wiener process, and an explicit formula for a (one-sided) linear boundary crossing probability for the Brownian bridge process to show that the one-sided boundary crossing probability $P\left(-\infty, g^{(n)}\right)$ for a piecewise-linear function $g^{(n)}$ can be represented as an $n$-fold Gaussian integral. Novikov et al. [12] gave, in their Theorem 1, a more general formula for the two-sided boundary crossing probabilities with arbitrary (measurable) boundaries $g_{ \pm}$. It is equivalent to the following representation: for any Borel set $B$,

$$
P\left(g_{-}, g_{+} ; B\right)=\mathrm{E}\left[\mathbf{1}_{\left\{W_{T} \in B\right\}} \prod_{i=0}^{n-1} p_{i}\left(g_{-}, g_{+} \mid W_{t_{i}}, W_{t_{i+1}}\right)\right],
$$

with $\mathbf{1}_{A}$ being the indicator function of the event $A$ and

$$
p_{i}\left(g_{-}, g_{+} \mid x_{i}, x_{i+1}\right):=\mathrm{P}\left(g_{-}(s)<W_{s}<g_{+}(s), s \in\left[t_{i}, t_{i+1}\right] \mid W_{t_{i}}=x_{i}, W_{t_{i+1}}=x_{i+1}\right) .
$$

(This representation also appeared in Theorem 2 of [14], with $B=\mathbb{R}$.)

In the special case when $g_{-}(s)=-\infty, s \in\left[t_{i}, t_{i+1}\right]$, and $g_{+}(s)$ is a linear boundary on this interval, the last probability has the following simple form, used in [20]:

$$
p_{i}\left(-\infty, g_{+} \mid x_{i}, x_{i+1}\right)=1-\exp \left\{-\frac{2\left(g_{+}\left(t_{i}\right)-x_{i}\right)\left(g_{+}\left(t_{i+1}\right)-x_{i+1}\right)}{t_{i+1}-t_{i}}\right\} .
$$

(This is a well-known expression for the linear boundary crossing probability for the Brownian bridge process - see, e.g. p. 63 of [2].) In the case of two-sided linear boundaries $g_{ \pm}$, the probability $p_{i}\left(g_{-}, g_{+} \mid x, y\right)$ is given by a rapidly convergent infinite series of exponential functions (for details and numerical examples, see, e.g. [12] or [14]). In both cases (i.e. of onesided and two-sided piecewise-linear boundaries), the complexity of the numerical computation of the $n$-fold Gaussian integral on the right-hand side of (7) appears to be acceptable, due to the relatively simple form of the functions $p_{i}\left(g_{-}, g_{+} \mid x_{i}, x_{i+1}\right)$.

Di Nardo et al. [7] found another parametric family of one-sided boundaries (that we call 'generalized Daniels' boundaries', cf. [5]), for which the probability $p_{i}\left(-\infty, g_{+} \mid x_{i}, x_{i+1}\right)$ also has a relatively simple form, as follows. If, for $t \in\left[t_{i}, t_{i+1}\right]$,

$$
y(t):=\left(d_{1}-d_{2}\right)\left(t-t_{i}\right)+d_{2}<u(t):=\left(d_{1}^{*}-d_{2}^{*}\right)\left(t-t_{i}\right)+d_{2}^{*}
$$

with $d_{1}, d_{2}, d_{1}^{*}, d_{2}^{*} \in \mathbb{R}$ and, on that time interval,

$$
g_{+}(t)=x_{i}+\frac{\left(t-t_{i}\right)\left(x_{i+1}-x_{i}\right)}{t_{i+1}-t_{i}}+u(t)-\frac{\left(t-t_{i}\right) \ln \left(\frac{1}{2} D(t)\right)}{2\left(u\left(t_{i}\right)-y\left(t_{i}\right)\right)}
$$


with

$$
D(t)=C_{1}+\sqrt{C_{1}^{2}+4 C_{2}^{2} \exp \left\{-4 \frac{(u(t)-y(t))\left(u\left(t_{i}\right)-y\left(t_{i}\right)\right)}{t-t_{i}}\right\}}>0,
$$

where $C_{1}, C_{2}>0$, then

$$
\begin{aligned}
p_{i}\left(-\infty, g_{+} \mid x_{i}, x_{i+1}\right)= & 1-C_{1} \exp \left\{-\frac{2 d_{1}^{*}\left(u\left(t_{i}\right)-y\left(t_{i}\right)\right)}{t_{i+1}-t_{i}}\right\} \\
& -C_{2} \exp \left\{-\frac{4\left(2 d_{1}^{*}-d_{1}\right)\left(u\left(t_{i}\right)-y\left(t_{i}\right)\right)}{t_{i+1}-t_{i}}\right\} .
\end{aligned}
$$

(Our notation is slightly different to that in [7].)

If $C_{1}=1$ and $C_{2}=0$, then the function $g_{+}(t)$ in (9) is linear and, by setting $d_{2}^{*}=g_{+}\left(t_{i}\right)-x_{i}$ and $d_{1}^{*}-d_{2}^{*}=g_{+}\left(t_{i+1}\right)-x_{i+1}$, we get, from (10), the well-known result (8). Generally, the parametric curve (9) depends on six parameters, so we can use it as a second-order spline with the boundary conditions

$$
g_{+}^{(n)}\left(t_{i}\right)=g_{+}\left(t_{i}\right), \quad \frac{\mathrm{d}}{\mathrm{d} t} g_{+}^{(n)}\left(t_{i}\right)=\frac{\mathrm{d}}{\mathrm{d} t} g_{+}\left(t_{i}\right), \quad t_{i}=\frac{i T}{n}, \quad i=0,1, \ldots, n .
$$

It is well known (see, e.g. Chapter 1 of [18]) that the approximation rate of four-times continuously differentiable functions $g_{ \pm}(t)$ by second-order spline functions $g_{ \pm}^{(n)}(t)$ on uniform partitions is

$$
\varepsilon:=\left\|g_{ \pm}-g_{ \pm}^{(n)}\right\|=O\left(n^{-4}\right) \quad \text { as } n \rightarrow \infty .
$$

Therefore, by Theorem 1 we will have, in this case,

$$
\left|P\left(g_{-}, g_{+} ; B\right)-P\left(g_{-}^{(n)}, g_{+}^{(n)} ; B\right)\right|=O\left(n^{-4}\right) .
$$

Of course, to satisfy the additional boundary conditions for such an approximation, we would have to solve a system of nonlinear equations and, therefore, the computational complexity of this approach could be greater than that of the piecewise-linear approximation.

In conclusion, note that, in the literature, there exist several quite different approaches to the computation of numerical approximations to $P\left(g_{-}, g_{+}\right)$and $P(-\infty, g)$, which have different computational complexities (see, e.g. [6], [7], [8], [9], [16], and references therein). Knowing not only the order, but also the form of the approximation error allows one to further improve the approximation rate by using the so-called Richardson extrapolation, which is based on the idea of extrapolating computed results to much larger values of $n$ (see, e.g. [1]).

Section 2 contains the proofs of the stated results. In Section 3, we will give the price of a double-barrier kick-out call option as a Black-Scholes-type formula involving two probabilities of the form $P\left(g_{-}, g_{+} ;(x, \infty)\right)$ and discuss an application of Theorem 1 to estimating the accuracy of approximations for double-barrier option prices.

\section{Proofs}

Due to the self-similarity property of the Wiener process, we can assume, without loss of generality, that $T=1$ (the general case bounds will then follow by the standard scaling argument). 


\subsection{Proof of Theorem 1}

We will begin the proof of Theorem 1 with the obvious relation

$$
P\left(g_{-}+\varepsilon, g_{+}-\varepsilon\right) \leq P\left(f_{-}, f_{+}\right) \leq P\left(g_{-}-\varepsilon, g_{+}+\varepsilon\right) .
$$

Note that

$$
\begin{aligned}
0 & \leq P\left(g_{-}-\varepsilon, g_{+}+\varepsilon\right)-P\left(g_{-}, g_{+}\right) \\
& =\left[P\left(g_{-}-\varepsilon, g_{+}+\varepsilon\right)-P\left(g_{-}, g_{+}+\varepsilon\right)\right]+\left[P\left(g_{-}, g_{+}+\varepsilon\right)-P\left(g_{-}, g_{+}\right)\right],
\end{aligned}
$$

where both terms on the right-hand side can be dealt with (with a view to establishing bounds on them) in the same way. It then suffices to consider, say, the second one only, and for that term we clearly have

$$
\begin{aligned}
P\left(g_{-}, g_{+}+\varepsilon\right)-P\left(g_{-}, g_{+}\right) & =\mathrm{P}\left(0 \leq \sup _{0 \leq t \leq 1}\left(W_{t}-g_{+}(t)\right)<\varepsilon, \inf _{0 \leq t \leq 1}\left(W_{t}-g_{-}(t)\right)>0\right) \\
& \leq \mathrm{P}\left(0 \leq \sup _{0 \leq t \leq 1}\left(W_{t}-g_{+}(t)\right)<\varepsilon\right) \\
& =P\left(-\infty, g_{+}+\varepsilon\right)-P\left(-\infty, g_{+}\right)=: D_{\varepsilon}\left(g_{+}\right) \\
& \leq \sup _{f \in \operatorname{Lip}_{K}} D_{\varepsilon}(f)=: D_{\varepsilon} .
\end{aligned}
$$

As the same argument also applies to the first term, we find that

$$
0 \leq P\left(g_{-}-\varepsilon, g_{+}+\varepsilon\right)-P\left(g_{-}, g_{+}\right) \leq 2 D_{\varepsilon} \text {. }
$$

Similarly,

$$
0 \leq P\left(g_{-}, g_{+}\right)-P\left(g_{-}+\varepsilon, g_{+}-\varepsilon\right) \leq 2 D_{\varepsilon},
$$

and, together with (11), these inequalities imply that

$$
\left|P\left(g_{-}, g_{+}\right)-P\left(f_{-}, f_{+}\right)\right| \leq 2 D_{\varepsilon} .
$$

Essentially the same argument shows that

$$
\left|P\left(g_{-}, g_{+} ; B\right)-P\left(f_{-}, f_{+} ; B\right)\right| \leq 2 D_{\varepsilon},
$$

as well. In the case of one-sided boundaries, an even simpler argument gives

$$
\left|P\left(-\infty, g_{+}\right)-P\left(-\infty, f_{+}\right)\right| \leq D_{\varepsilon} .
$$

The desired bounds (2) and (3) now follow from the next assertion.

Lemma 1. Let $g$ be a function on $[0,1]$, with $g(0) \geq 0$, such that, for some $K^{ \pm} \in[0, \infty)$,

$$
-K^{-} h \leq g(t+h)-g(t) \leq K^{+} h, \quad 0 \leq t<t+h \leq 1 .
$$

Then

$$
D_{\varepsilon}(g) \leq\left(2 K^{+}+0.5 K^{-}+2\right) \varepsilon, \quad \varepsilon>0 .
$$


Proof. Let $\tau:=\inf \left\{t>0: W_{t}>g(t)\right\}$ and observe that

$$
\begin{aligned}
D_{\varepsilon}(g) & =\mathrm{P}\left(0 \leq \sup _{0 \leq t \leq 1}\left(W_{t}-g(t)\right)<\varepsilon\right) \\
& =\int_{0}^{1} \mathrm{P}(\tau \in \mathrm{d} t) \mathrm{P}\left(\sup _{t \leq s \leq 1}\left(W_{s}-g(s)\right)<\varepsilon \mid W_{t}=g(t)\right) \\
& \leq \int_{0}^{1} \mathrm{P}(\tau \in \mathrm{d} t) \mathrm{P}\left(\sup _{0 \leq s \leq 1-t}\left(W_{s}-K^{+} s\right)<\varepsilon\right) .
\end{aligned}
$$

The last probability is known in explicit form (see, e.g. Equation 1.1.4 on p. 197 of [2]): denoting by $\Phi$ the standard normal distribution function, we get

$$
\begin{aligned}
& \mathrm{P}\left(\sup _{0 \leq s \leq 1-t}\left(W_{s}-K^{+} s\right)<\varepsilon\right) \\
& \quad=\Phi\left(K^{+} \sqrt{1-t}+\frac{\varepsilon}{\sqrt{1-t}}\right)-\mathrm{e}^{-2 K^{+} \varepsilon} \Phi\left(K^{+} \sqrt{1-t}-\frac{\varepsilon}{\sqrt{1-t}}\right) \\
& \quad \leq \Phi\left(K^{+} \sqrt{1-t}+\frac{\varepsilon}{\sqrt{1-t}}\right)-\Phi\left(K^{+} \sqrt{1-t}-\frac{\varepsilon}{\sqrt{1-t}}\right)+\left(1-\mathrm{e}^{-2 K^{+} \varepsilon}\right) .
\end{aligned}
$$

Using the obvious inequalities $\Phi^{\prime}(x) \leq(2 \pi)^{-1 / 2}$ and $1-\mathrm{e}^{-2 K^{+} \varepsilon} \leq 2 K^{+} \varepsilon$, we see from this representation that the probability on the left-hand side does not exceed $(2 / \pi(1-t))^{1 / 2} \varepsilon+$ $2 K^{+} \varepsilon$.

We next consider $\mathrm{P}(\tau \in \mathrm{d} t)$. For any fixed $t \in[0,1]$, introduce the boundary

$$
g_{t}(s):=g(t)+K^{-}(t-s), \quad 0 \leq s \leq 1,
$$

and let

$$
\tau_{t}:=\inf \left\{s>0: W_{s}>g_{t}(s)\right\}=\inf \left\{s>0: W_{s}+K^{-} s>g(t)+K^{-} t\right\} .
$$

Obviously, due to our assumption (12),

$$
\mathrm{P}(\tau \in(t, t+h)) \leq \mathrm{P}\left(\tau_{t} \in(t, t+h)\right), \quad 0 \leq t<t+h \leq 1,
$$

and, hence, it only remains to bound the right-hand side of this inequality.

Since $\left\{W_{s}+K^{-} s\right\}_{s \geq 0}$ is a continuous processes with stationary, independent increments, the distribution of $\tau_{t}$ can be readily found from Kendall's formula (see, e.g. Theorem 1 on p. 66 of [3]; see also Equation 2.0.2 on p. 223 of [2] for our special case): it will have the density

$$
v_{t}(s):=\frac{\mathrm{P}\left(\tau_{t} \in \mathrm{d} s\right)}{\mathrm{d} s}=\frac{g(t)+K^{-} t}{\sqrt{2 \pi s^{3}}} \exp \left\{\frac{-\left(g(t)+K^{-}(t-s)\right)^{2}}{2 s}\right\}, \quad s>0 .
$$

From (15), it follows that $\tau$ will have a density $p(t)$ such that

$$
p(t) \leq v_{t}(t), \quad 0<t<1 .
$$


Returning to (13) and our bound for the integrand contained in its last line, we have

$$
\begin{aligned}
D_{\varepsilon}(g) & \leq \varepsilon \sqrt{\frac{2}{\pi}} \int_{0}^{1} \frac{p(t) \mathrm{d} t}{\sqrt{1-t}}+2 K^{+} \varepsilon \int_{0}^{1} p(t) \mathrm{d} t \\
& \leq \varepsilon \sqrt{\frac{2}{\pi}}\left(\int_{0}^{1 / 2}+\int_{1 / 2}^{1}\right) \frac{p(t) \mathrm{d} t}{\sqrt{1-t}}+2 K^{+} \varepsilon \\
& \leq\left[\frac{2}{\sqrt{\pi}} \int_{0}^{1 / 2} p(t) \mathrm{d} t+\sqrt{\frac{2}{\pi}} \int_{1 / 2}^{1} \frac{v_{t}(t) \mathrm{d} t}{\sqrt{1-t}}+2 K^{+}\right] \varepsilon .
\end{aligned}
$$

The assertion of the lemma (and, hence, that of Theorem 1) is now immediate from the obvious inequalities $\int_{0}^{1 / 2} p(t) \mathrm{d} t<1$ and $2 \pi^{-1 / 2}+2^{-1 / 2}<2$, and the following bound.

Lemma 2. Under the assumptions of Lemma 1,

$$
\sqrt{\frac{2}{\pi}} \int_{1 / 2}^{1} \frac{v_{t}(t) \mathrm{d} t}{\sqrt{1-t}} \leq \frac{K^{-}}{2}+\frac{1}{\sqrt{2}}
$$

Proof. First we will find the maximum possible value of $v_{t}(t)$ over all admissible values $g(t) \geq g(0)-K^{-} t \geq-K^{-} t$ (as $g(0) \geq 0$ and (12) holds). To this end, we compute

$$
\sup \left\{m(y):=\left(y+K^{-} t\right) \mathrm{e}^{-y^{2} / 2 t}: y \geq g(0)-K^{-} t\right\}
$$

by taking the derivative of the function $m(y)$ with respect to $y$ and equating it to zero, which yields

$$
0=1-\left(y+K^{-} t\right) y / t
$$

Solving this for $y$, we get

$$
y_{ \pm}=-\frac{1}{2} K^{-} t \pm \sqrt{t+\frac{1}{2} K^{-} t} .
$$

Now, noting that the root $y_{-}$is inadmissible and that $m^{\prime \prime}\left(y_{+}\right)<0$, we see that $m\left(y_{+}\right)$is indeed a maximum, so that

$$
v_{t}(t) \leq \frac{y_{+}+K^{-} t}{\sqrt{2 \pi t^{3}}}=\frac{1}{\sqrt{2 \pi}}\left(\frac{K^{-}}{2 \sqrt{t}}+\sqrt{\frac{1}{t^{2}}+\frac{\left(K^{-}\right)^{2}}{4 t}}\right) \leq \frac{1}{\sqrt{2 \pi}}\left(\frac{K^{-}}{\sqrt{t}}+\frac{1}{t}\right),
$$

using $\left(a^{2}+b^{2}\right)^{1 / 2} \leq a+b$ for $a, b \geq 0$. Therefore, with $r=\frac{1}{2}$,

$$
\begin{aligned}
\sqrt{\frac{2}{\pi}} \int_{r}^{1} \frac{v_{t}(t) \mathrm{d} t}{\sqrt{1-t}} & \leq \frac{1}{\pi}\left[\int_{r}^{1} \frac{K^{-} \mathrm{d} t}{\sqrt{t(1-t)}}+\int_{r}^{1} \frac{\mathrm{d} t}{t \sqrt{1-t}}\right] \\
& =\frac{1}{\pi}\left[K^{-}(\pi-2 \arcsin \sqrt{r})+2 \int_{\arcsin \sqrt{r}}^{\pi / 2} \frac{\mathrm{d} u}{\sin u}\right] \\
& \leq \frac{K^{-}}{\pi}(\pi-2 \arcsin \sqrt{r})+\frac{2\left(\frac{1}{2} \pi-\arcsin \sqrt{r}\right)}{\pi \sqrt{r}} \\
& =\frac{K^{-}}{2}+\frac{1}{\sqrt{2}} .
\end{aligned}
$$

This completes the proofs of Lemmas 1 and 2 and, therefore, that of Theorem 1. 


\subsection{Proof of Corollary 1}

To prove Corollary 1, it suffices to show that, for the maximum deviation of the piecewiselinear approximant $f$ from the original boundary function $g$ with the assumed properties, we obtain

$$
\|f-g\| \leq \frac{1}{8} \gamma \delta^{2}
$$

as the desired result will then immediately follow from Theorem 1. The proof of bound (16) is elementary and is included only for the sake of completeness.

Clearly,

$$
\|f-g\|=\max _{0<i \leq n} \max _{t \in\left[t_{i-1}, t_{i}\right]}|f(t)-g(t)|,
$$

so we have to show that the bound holds for the maximum deviation on each of the subintervals $\left[t_{i-1}, t_{i}\right], i=1, \ldots, n$. Consider the first of these - the same argument will clearly work for all the others. Let

$$
\xi=\arg \max _{t \in\left[t_{0}, t_{1}\right]}|f(t)-g(t)|
$$

and observe that $g^{\prime}(\xi)=f^{\prime}(\xi)$. By setting

$$
h(t)=g(\xi)+g^{\prime}(\xi)(t-\xi), \quad t \in\left[t_{0}, t_{1}\right],
$$

we see from this observation that the plots of the functions $f$ and $h$, which are linear (at least on the subinterval $\left.\left[t_{0}, t_{1}\right]\right)$, are parallel to one another, i.e. $f(t)-h(t)=$ const, $t \in\left[t_{0}, t_{1}\right]$.

Next, for $j=0,1$, we have

$$
\begin{aligned}
\max _{t \in\left[t_{0}, t_{1}\right]}|f(t)-g(t)| & =|f(\xi)-g(\xi)|=|f(\xi)-h(\xi)|=\left|f\left(t_{j}\right)-h\left(t_{j}\right)\right| \\
& =\left|g\left(t_{j}\right)-h\left(t_{j}\right)\right|=\left|g\left(t_{j}\right)-g(\xi)-g^{\prime}(\xi)\left(t_{j}-\xi\right)\right| \\
& =\left|\int_{\xi}^{t_{j}}\left[\int_{\xi}^{u} g^{\prime \prime}(v) \mathrm{d} v\right] \mathrm{d} u\right| \\
& \leq \frac{1}{2} \gamma\left(t_{j}-\xi\right)^{2} .
\end{aligned}
$$

Now, since $\min _{j=0,1}\left\{\left(t_{j}-\xi\right)^{2}\right\} \leq\left(\frac{1}{2}\left(t_{1}-t_{0}\right)\right)^{2} \leq \frac{1}{4} \delta^{2}$, our bound (16) and, hence, the statement of Corollary 1 are proved.

\section{Approximations for time-dependent barrier options prices}

In this section, we will discuss how the above results can be applied to barrier options pricing.

It is well known that, under the no-arbitrage assumption, the fair price of a (replicable) option (on an underlying asset with a price process $\left\{S_{t}\right\}_{t \geq 0}$ ) with maturity $T$ and payoff $X_{T}$ is given by $\mathrm{E}\left(X_{T} / B_{T}\right)$, where $\mathrm{E}$ denotes the operation of taking expectation with respect to a risk-neutral measure $\mathrm{P}$ and $\left\{B_{t}\right\}_{t \geq 0}$ is the bank account process (for details, see, e.g. [19]). Consider a kick-out barrier call option with strike $K_{T}$ and time-dependent lower and upper barriers $G_{ \pm}(t)$ such that $G_{-}(t)<G_{+}(t), t \leq T$. In this case, the payoff function is given by

$$
X_{T}=\left(S_{T}-K_{T}\right) \mathbf{1}_{\left\{S_{T}>K_{T} ; G_{-}(t)<S_{t}<G_{+}(t), t \in[0, T]\right\}} .
$$

Assume that the bank account process is nonrandom and has the form

$$
B_{t}=\exp \left\{\int_{0}^{t} r_{s} \mathrm{~d} s\right\},
$$


where $r_{t}$ is a positive function of time (the spot interest rate). Under the assumptions of a Black-Scholes-type diffusion model with a term structure assuming a variable deterministic volatility $\sigma_{t}>0$ with $\sigma^{2}:=\int_{0}^{T} \sigma_{t}^{2} \mathrm{~d} t<\infty$, the price of the underlying asset $S_{t}$ has, under the risk-neutral measure, the following representation:

$$
S_{t}=S_{0} \exp \left\{\int_{0}^{t}\left[r_{s}-\frac{1}{2} \sigma_{s}^{2}\right] \mathrm{d} s+\int_{0}^{t} \sigma_{s} \mathrm{~d} W_{s}\right\} .
$$

It is quite obvious that, by using the 'natural time change' (with the new 'time' being $t^{\prime}:=$ $\sigma^{-2} \int_{0}^{t} \sigma_{s}^{2} \mathrm{~d} s$ ), we can make the volatility constant over the time interval $[0, T]$. For simplicity's sake, let us just assume that we have already done so, thus yielding, in the natural time scale, the representation

$$
S_{t}=S_{0} \exp \left\{\int_{0}^{t}\left[r_{s}-\frac{1}{2} \sigma^{2}\right] \mathrm{d} s+\sigma W_{t}\right\} .
$$

(We retain the same notation for the time variable $t$ and also for the respectively transformed interest rate $r_{t}$, barrier functions $G_{ \pm}(t)$, and the Wiener process $W_{t}$; the interested reader could easily do all the calculations by himself.)

The following statement gives a representation of the option price in terms of the boundary crossing probabilities. Observe that - for a clear reason - the pricing formula has the same structure as the Black-Scholes formula for the European call and, hence, could be considered to be a natural generalization of the latter. Thus, whereas to price a European call it suffices to find the values of two normal probabilities, to price a barrier call one simply has to find the values of two probabilities of the form $P\left(g_{-}, g_{+},(x, \infty)\right)$.

Proposition 1. The fair price of the above double-barrier call option under model (17) is given by

$$
S_{0} p_{1}-K_{T} \exp \left\{-\int_{0}^{T} r_{s} \mathrm{~d} s\right\} p_{0},
$$

where

$$
\begin{aligned}
& p_{1}=\mathrm{P}\left(f_{-}(t)<\sigma W_{t}+\sigma^{2} t<f_{+}(t), t \in[0, T] ; \sigma W_{T}+\sigma^{2} T>F\right), \\
& p_{0}=\mathrm{P}\left(f_{-}(t)<\sigma W_{t}<f_{+}(t), t \in[0, T] ; \sigma W_{T}>F\right), \\
& F=\ln \left(K_{T} / S_{0}\right)+\frac{1}{2} \sigma^{2} T-\int_{0}^{T} r_{S} \mathrm{~d} s,
\end{aligned}
$$

and

$$
f_{ \pm}(t)=\ln \left(G_{ \pm}(t) / S_{0}\right)+\frac{1}{2} \sigma^{2} t-\int_{0}^{t} r_{s} \mathrm{~d} s, \quad t \in[0, T] .
$$

One can easily prove this statement using Girsanov's transformation (for details in the case of a one-sided barrier, see, e.g. [13]).

To calculate the probabilities $p_{0}$ and $p_{1}$, one could use several different techniques: a PDE approach [21], integral equations for the case of one-sided barriers [7], [16], or Monte Carlo simulation [15]. As both probabilities $p_{0}$ and $p_{1}$ are of the form $P\left(g_{-}, g_{+} ;(x, \infty)\right)$, one could also use a numerical approximation based on the integral representation (7), with properly chosen spline approximations and respective probabilities $p_{i}\left(g_{-}, g_{+} \mid x_{i}, x_{i+1}\right)$. In particular, using boundary approximations that are piecewise linear (on uniform partitions) will yield, by Corollary 1, an approximation rate of the order $O\left(n^{-2}\right)$. Using generalized Daniels' boundaries 
(see Remark 6, above), the rate of convergence could potentially be improved to $O\left(n^{-4}\right)$ (or to an even higher order). However, a discussion of the computational aspects and efficiencies of different numerical techniques is beyond the scope of the present paper.

\section{Acknowledgements}

This research was supported by the ARC Centre of Excellence for Mathematics and Statistics of Complex Systems and ARC Large Grant A0010474. The authors are grateful to the referee for his useful comments, which helped to improve the paper.

\section{References}

[1] Acton, F. S. (1990). Numerical Methods that Work. Mathematical Association of America, Washington, DC.

[2] Borodin, A. N. and SAlminen, P. (2002). Handbook of Brownian Motion-Facts and Formulae, 2nd edn. Birkhäuser, Basel.

[3] Borovkov, A. A. (1976). Stochastic Processes in Queueing Theory. Springer, New York.

[4] Borovkov, K. A. (1982). Limit theorems for some types of random walks. Doctoral Thesis, Steklov Mathematical Institute, Moscow.

[5] Daniels, H. E. (1969). The minimum of a stationary Markov process superimposed on a U-shaped trend. $J$. Appl. Prob. 6, 399-408.

[6] Daniels, H. E. (1996). Approximating the first crossing-time density for a curved boundary. Bernoulli 2, 133143.

[7] Di Nardo, E., Nobile, A. G., Pirozzi, E. and Ricciardi, L. M. (2001). A computational approach to firstpassage-time problems for Gauss-Markov processes. Adv. Appl. Prob. 33, 453-482.

[8] Durbin, J. (1992). The first-passage density of the Brownian motion process to a curved boundary. J. Appl. Prob. 29, 291-304.

[9] Lerche, H. R. (1986). Boundary Crossing of Brownian Motion (Lecture Notes in Statistics 40). Springer, Berlin.

[10] Nagaev, S. V. (1970). The rate of convergence in a certain boundary value problem. I. Theory Prob. Appl. 15, 179-199.

[11] Nagaev, S. V. (1970). The rate of convergence in a certain boundary value problem. II. Theory Prob. Appl. 15, 419-444.

[12] Novikov, A. A., Frishling, V. and Kordzakhia, N. (1999). Approximations of boundary crossing probabilities for a Brownian motion. J. Appl. Prob. 36, 1019-1030.

[13] Novikov, A. A., Frishling, V. And Kordzakhia, N. (2003). Time-dependent barrier options and boundary crossing probabilities. Georgian Math. J. 10, 325-334.

[14] Pötzelberger, K. and Wang, L. (2001). Boundary crossing probability for Brownian motion. J. Appl. Prob. 38, 152-164.

[15] Roberts, G. O. and Shortland, C. F. (1997). Pricing barrier options with time-dependent coefficients. Math. Finance 7, 83-93.

[16] Sacerdote, L. and Tomassetti, F. (1996). On evaluations and asymptotic approximations of first-passage-time probabilities. Adv. Appl. Prob. 28, 270-284.

[17] Sahanenko, A. I. (1974). On the rate of convergence in a boundary value problem. Theory Prob. Appl. 19, 416-421.

[18] Shikin, E. V. And Plis, A. I. (1995). Handbook on Splines for the User. CRC Press, Boca Raton, FL.

[19] Shiryaev, A. N. (1999). Essentials of Stochastic Finance: Facts, Models, Theory. World Scientific, Singapore.

[20] Wang, L. ANd Pötzelberger, K. (1997). Boundary crossing probability for Brownian motion and general boundaries. J. Appl. Prob. 34, 54-65.

[21] Wilmott, P., Dewynne, J. And Howison, S. (1995). Option Pricing: Mathematical Models and Computation. Cambridge Financial Press. 